\title{
Exploration and Practice of Classified Cultivation Concept of "Harmonious without Uniformity" for Graduate Students in the School of Electronic Engineering
}

\author{
Cao Yang, Li Shixin* \\ School of Electronic Engineering, Tianjin Vocational and Technical Normal University \\ 742116457@qq.com
}

\begin{abstract}
Guided by the idea of "harmonious without uniformity", targeting at the academic postgraduate and professional postgraduate students of 2017 session in the School of Electronic Engineering, this paper formulates the classified cultivation objectives, focusing on the improvement of scientific research and innovation ability of academic degree graduates, and the progress of professional practice ability of professional degree graduates. Different curriculum systems are established to provide different research or practice platforms for students, thereby meeting the social demand for electronic engineering personnel, and improving the professional quality of graduate students in electronic engineering.
\end{abstract}

Keywords-Harmonious but different; School of Electronic Engineering; Academic postgraduate; Professional postgraduate; Classified cultivation

\section{INTRODUCTION}

The Confucianism founder Confucius has a well-known saying: "The gentleman is harmonious but different, while a base person is the same with no harmony." It means that in our life, the right way is to refuse similarity and reach a consensus in mutual debate and argument. In ancient China, the idea of "Harmony but different" is also an important magic tool to deal with the relationship between different academics, thoughts, groups and different cultures. It is the internal motivation, the necessary path and the basic law of academic development

In today's society, practical ability is obtaining more and more attention, and the professional masters are becoming more and more common. The number of professional masters in our school is also increasing year by year. Since the purpose of setting up degrees of academic graduate and professional graduate is different, therefore, for colleges and universities, how to conduct classified cultivation and train these two types of graduate students to meet the requirements of the state for the cultivation of graduate students is a problem we must seriously consider now [1].

Radically speaking, the establishment of academic graduate cultivation is aimed at academic research, basic theoretical innovation and the application of theory. The main purpose of training is to cultivate research-oriented talents to meet the needs of basic research talents in various industries of today's society. The establishment of professional graduate cultivation is aimed at cultivating students' application of professional ability and technological innovation. It mainly cultivates application-oriented talents to meet the needs of professional talents in various industries of today's society. Therefore, the training objectives of the two are different, and as a training side, the school is necessary to make divisions in the graduate training program and the curriculum. Otherwise, it is difficult to achieve the quality goal for talent training [2-3]. Therefore, when training academic and professional masters at the same time, as a training unit, classified cultivation is the only path.

From the current status quo, for the training programs of academic masters and professional masters, most colleges simply add or subtract the contents, and the training of professional masters also copies or follows the original academic master training program.[4] This is obviously not suitable. Academic and professional masters have distinct characteristics in both the cultivating objectives and the process of cultivation, even though it is the same college, the same major, or even cultivated by the same tutor. It cannot be done through a simple programs addition or subtraction. Therefore, for cultivating masters in the current colleges and universities, how to make the two types of students to achieve "harmony but different" is of great importance, and classified cultivation is an irresistible trend and also an inevitable choice.

\section{THE NECESSITY OF CLASSIFIED CULTIVATION}

\section{A. Classified cultivation for graduate students is needed for educational development}

After more than 30 years of reform and development, China's graduate education has basically reached the strategic goal of self-cultivating high-level talents, and has established a graduate education system with complete disciplines and diverse training types. By the end of 2103 , there were nearly 1.8 million graduate students in China, including about 1.5 million master students, making our country a large education country [5]. However, with the expansion of enrollment and the increase and change of social needs, the current graduate

Project Funding: School-level project funding. 
education mode has become a bottleneck restricting the development of graduate education.

\section{B. Classified cultivation for graduate students needs to be performed to improve education efficiency}

In recent years, academic graduate students in the graduate enrollment system in China accounts for $95 \%$, however, less than $10 \%$ of them are able to engage in related academic or theoretical research after leaving the school, and in terms of social employment, academic graduate students are relatively weak compared with professional graduate students. In recent years, most of the postgraduate students in China have entered the non-traditional academic field. Their employment intention and social positioning have been gradually transferred to enterprises and institutions to undertaken professional work requiring certain academic standard. Therefore, the classified cultivation of academic and professional masters is the current need for improving the efficiency of education.

\section{Classified cultivation for graduate students needs to be performed to adapt to students' development}

In recent years, graduate examinations have become an increasingly popular choice for college graduates, but in fact, students have different purposes for their master's career. Some of them hope to get better employment opportunities and higher salary after master graduation. The other part of them hope to be able to continue depth study of doctor's degree after the postgraduate study, to give play to their own ability in the future in the field of scientific research, and to conduct scientific experiments and theoretical research. There are even some students who simply don't want to graduate and want to continue their campus life. For different students, the goals or motivations are also different in the graduate period. Thus, the attitude and actions of receiving education are different, so that the content and methods of education should be different. Therefore, classified cultivation and targeted cultivation will better adapt to the different orientations of graduate students to meet the needs of students' personal development.

\section{SPECIFIC MEASURES FOR CLASSIFIED CULTIVATION}

\section{A. Classified targeting of cultivation goals}

Targeting cultivation goals is the key to classified cultivation. First of all, the cultivating unit must have a correct understanding to the similarities and differences between academic and professional masters. In seeking common ground while reserving differences, the cultivating unit must guide the actual classification and cultivation work according to the different national standards for the award of the two degrees and the educational goals training unit wants to achieve.

Academic degrees are established according to different disciplines, be research-oriented, biased in theory, trains researchers and theoretical analysts; professional degrees are practice-oriented, emphasizes on competence practice and technology application, training application-oriented talents who have received formal training in technology and have strong practical ability.
The academic postgraduate training program pays more attention to the cultivation of disciplinary theory and theoretical literacy, focuses on students' basic theoretical education and systematic research and training, improving scientific research ability and academic innovation ability, and cultivating academic research talents. The professional postgraduate training program pays more attention to the cultivation of students' practical ability and learning relevant professional skills, advocates the combination of production, learning and research, increases the proportion of cases in the case teaching method, strengthens the practice base construction, and cultivates application-oriented technical talent.

\section{B. Classified establishment of two different curriculum systems}

For a graduate student, in order to have a solid theoretical foundation and complete professional knowledge, as well as the knowledge structure required for scientific research and discipline work in his/her own field, the curriculum setting is the main link. How the curriculum is set up reflects the knowledge structure in the disciplinary field. The graduates of academic degrees and professional degrees should learn different knowledge and skills during the study period, so the curriculum setting should also be different.

The academic graduate curriculum setting should be guided by "scientific research", and adopt the method of combining the responsibility of tutoring teachers with the collective training of discipline. It should not only play the leading role of the tutor in training, but also pay attention to the collective strength of the discipline. In the whole process of cultivation, a combination of theoretical study and scientific research is required. It is necessary for graduate students to master the basic theoretical knowledge and professional skills of the discipline, and also to master the research methods in the scientific field and have the ability to conduct academic research and experiments independently.

The establishment of professional postgraduate curriculum should be guided by "practical ability", with technical capabilities as the goal, and with the improvement of comprehensive quality and application technology capabilities as the core. The teaching content should emphasize the organic combination of research and practice, highlighting case teaching and practical teaching; in the teaching process, we should pay attention to methods of typical cases, on-site simulation, practical training and others. It is necessary to focus on cultivating the sense and ability of graduate students' dealing with practical problems.

\section{Establishing classified cultivating platform}

For academic graduate students, the training platform of "academic research" should be mainly established in the process of training, which mainly cultivates students' theoretical research level and scientific research and innovation ability. During the platform training, graduate students are required to carry out relevant academic research, academic reports, academic exchanges and other activities according to 
their respective research directions and professional level, and attend academic conferences frequently.

For the training of professional degree graduates, the training platform of "ability practice" should be mainly established in the process of training. In the process of training, it must adhere to the tenet of facing the needs of society and persisting in the cultivation of quality first, and strive to study and explore the training mode of production, education and research integration. We also should make full use of the effective resources of schools and society to cultivate "highlevel, practical, compound and international" engineering and technical talents and education talents in the field of electronic information.

\section{Adopting classified tutoring methods}

For academic graduate students, we should adopt a tutoring approach that emphasizes the improvement of "academic level", choose teachers with rich teaching experience and rich scientific research results to serve as academic masters' tutors, improve students' basic theoretical knowledge and academic innovation research ability. Within the college, we should set up a graduate tutor guidance team to strengthen communication and complement between the tutors in the discipline direction and research direction. Graduate students can also join the team to learn to solve problems, analyze problems, follow the mentor to contact new technologies, and improve academic innovation ability.

For professional graduates, they should adhere to the "professional quality" guidance method, not only to improve students' theoretical knowledge, but also to cultivate students' professional intervention ability. Therefore, it adopts the dualtutor system inside and outside the school, that is, the graduate instructors with engineering experience in the school and enterprise experts will jointly serve as tutors. The tutors in school is the base, tutors outside school should participate in the guidance work of practice, project research, courses and papers, and other aspects. The tutors should teach and educate with a high sense of responsibility, make strict requirement and ensure the quality of training, and urge the students to study the course, guide the selection of the thesis, consult the literature, research, engineering practice, thesis writing and defense according to the training plan. At the same time, through the postgraduate practice base established by the school, or the practice unit that students connected by themselves, go deep into the enterprise and combine the advantages of the school theory with the advantages of enterprise engineering practice.

\section{CUlTIVATING PROGRAM DESIGN}

Under the idea of "Harmonious but different", we set different research directions for the two: signal and system direction and information technology direction.

The academic master's cultivating program is divided into four major parts: degree, compulsory, elective and practice courses. Among which, the degree courses include theoretical and practical research on socialism with Chinese characteristics, English, An introduction to Dialectics of Nature, numerical analysis, matrix theory, educational scientific research methods, information theory and coding, digital image processing, and advanced electromagnetic fields. Compulsory courses include academic reports and professional foreign languages (translation of foreign language documents). Elective courses include optical communication technology, wavelet theory and applications, communication network protocols, and mathematical physics methods. Students choose three courses among them to study. The practical course consists of a fiveweek professional skills practice, a two-week e-course comprehensive practice, and a two-month engineering practice.

The professional master's cultivating program is also generally divided into four parts: the basic degree course, the compulsory degree course, the elective degree course and the practice course. Among which, the basic degree courses include ideological and political education, English, principles of education, curriculum and teaching theory, educational research methods, and psychological development and education for adolescents. Professional compulsory courses include information technology professional curriculum development and teaching material analysis, information technology professional teaching design and case analysis, vocational and technical education measurement and evaluation, and information technology professional development frontier topics. Professional elective courses include vocational education information, class teacher and class management, vocational education policies and regulations, mobile software development skills, communication network protocols, digital image processing, and DSP design and development. For professional postgraduate students, the practice course is undoubtedly the most important one. Therefore, for the practice course, we have arranged a five-week comprehensive experiment on information electronics technology, a three-month educational internship and a three-month corporate practice. We must strive to perfect the professional practice ability of professional masters.

\section{CONCLUSION}

In view of the differences between the academic and professional masters in the training ideas and training methods, this paper regards the training objectives and degree awarding standards as the fundamental basis for the design of the curriculum system, and continuously explores and practices according to the principle of "harmonious but different". In this paper, a set of training programs based on the concept of classified training for the academic graduate students in the direction of signal and system and professional graduate students in the direction of information technology has been established in the School of Electronic Engineering, and different tutors' guiding programs have been set up, different classified training platforms are established, and it will conduct tracking and evaluating in the new session of the graduate students. 


\section{REFERENCES}

[1] Zhang Leping, Wang Yingmi, Chen Xiaoping. Research on the recognition degree of full-time professional master training model[J]. Graduate Education Research, 2013

[2] Ministry of Education, National Development and Reform Commission, Ministry of Finance. Opinions on deepening postgraduate education reform. Teaching and Research [2013] No. 1, 2013-03-29.

[3] Zhang Leping, Wang Yingmi, Chen Xiaoping. Investigation and analysis of the training status of full-time engineering postgraduate students [J]. Degree and postgraduate education, 2012.

[4] Ji Cheng. Reconstruction and exploration of master's degree training program under the perspective of classified cultivation [J]. Higher Education Exploration, 2015.

[5] China Graduate Education Quality Annual Report (2014) [M]. Beijing: China Science and Technology Press, 2015

[6] Wang Jianhua, Tang Jianrong, Wu Linhai Research on the Differential Cultivation Model of Graduate Students Based on Classified Promotion[J]. Graduate Education Research, 2014. 